\title{
Fatigue Crack Paths in Coarse-Grained Magnesium
}

\author{
G. Nicoletto ${ }^{1}$, R. Konecna ${ }^{2}$ and A. Pirondi ${ }^{1}$ \\ ${ }^{1}$ Dept. of Industrial Engineering - University of Parma \\ Parco Area delle Scienze, 181/A - 43100 Parma - Italy \\ ${ }^{2}$ Dept. of Materials Engineering - University of Žilina \\ Velky Diel - 01100 Žilina - Slovak Republic \\ e-mail: nick@me.unipr.it; radomila_konecna@kmi.utc.sk; pirondia@me.unipr.it,
}

\begin{abstract}
This paper examines fatigue crack paths in coarse-grained magnesium alloys. Visualization of the microstructure by etching and continuous crack monitoring with a microscope provides evidence of the crack-grain interaction at different fatigue crack growth regimes. Near-threshold fatigue crack growth promotes the development of rough surfaces by activation of single slip mechanisms and crack deflection. The R-ratio effect on nearthreshold results is discussed in the light of the partial crack closure model. The role of grain boundaries and grain-to-grain crack deflections on fatigue crack growth rates is determined.
\end{abstract}

\section{INTRODUCTION}

Fatigue crack propagation of long cracks is customarily divided in three regimes, [1]. Regime A or near-threshold is of great practical importance and it is characterized by complex testing procedures and by the influence of many experimental and material factors. Regime B or Paris's regime has been extensively studied because of its usefulness for the damage tolerant approach to the fatigue design of aerospace structures. Regime $\mathrm{C}$ characterizes the rapid crack extension to final fracture. The main characteristics that differentiate Regimes A and B are summarized in Tab.1. It is noted that near-tip plasticity and its relationship with a typical material microstructural feature, such as the average grain size, can be used to discriminate between Regimes A and B.

Table 1. Characteristics of the FCG regimes (partially after [1]).

\begin{tabular}{|l|c|c|}
\hline & $\begin{array}{c}\text { Regime A } \\
\text { (Near-threshold) }\end{array}$ & $\begin{array}{c}\text { Regime B } \\
\text { (Paris's regime) }\end{array}$ \\
\hline Growth rates & Low growth rates & Intermediate growth rates \\
\hline Microscopic failure mode & Stage I, single shear & Stage II (striation) and duplex slip \\
\hline Fracture surface features & Faceted or serrated & Planar with ripples \\
\hline Crack closure levels & High & Low \\
\hline Microstructural effects & Large & Small \\
\hline Load ratio effects & Large & Small \\
\hline Near-tip plasticity & $\mathrm{r}_{\mathrm{c}}<\mathrm{d}_{\mathrm{g}}$ & $\mathrm{r}_{\mathrm{c}}>\mathrm{d}_{\mathrm{g}}$ \\
\hline
\end{tabular}


The near-threshold regime in metals is generally associated with a crack tip plasticity largely confined to select crystallographic planes with a reversed-shear mode of growth. Crack deflections due to microstructural heterogeneity can lead to mixed-mode displacements on the microscopic level and a faceted fracture surface. These displacements cause mismatch between upper and lower crack faces which in turn results in a positive closure load.

Here attention is devoted to the roughness-induced crack closure (RICC) because it is strongly influenced by the material microstructure and it is associated to a zigzag crack pattern. According to [1], RICC is promoted by: i) low stress intensity factor levels where the plastic zone, $r_{c}$, is smaller than the average grain diameter, $d_{g}$; ii) small crack opening displacements (i.e. low $\Delta \mathrm{K}$ and low R-ratios) of a size comparable to surface asperities; iii) coarse grained microstructures; iv) periodic deflections of the crack due to grain boundaries, second-phase particles and composite reinforcement; v) enhanced slip irreversibility.

This paper examines fatigue crack paths in coarse-grained magnesium alloys. Visualization of the microstructure by etching and continuous monitoring of the fracture path with a microscope provides evidence of the crack-grain interaction at different fatigue crack growth regimes. Finally, near-threshold fatigue crack growth test results are discussed in the light of the partial crack closure model of [2].

\section{EXPERIMENTAL DETAILS}

Magnesium alloys have a great potential as construction materials for their excellent strength/density ratio and for their good casting and machinability properties, [3]. The materials considered in this paper are pure magnesium and the Mg-alloy AZ91. The chemical compositions of the latter is (in mass \%) Al: 8.03, Zn: 0.53, Mn: 0.18, Si: 0.064, Cu: 0.035, Fe: 0.012, Be: 0.0004, Mg: balance. The materials were cast and delivered after a T6 heat treatment, [3], and were characterized by a rather coarse microstructure with average grain size $\mathrm{d}_{\mathrm{g}}=300 \mu \mathrm{m}$ for AZ91 and even bigger, $\mathrm{d}_{\mathrm{g}}=800 \mu \mathrm{m}$, for pure $\mathrm{Mg}$.

Fatigue crack growth experiments using small single-edge-notched specimens were performed at $15 \mathrm{~Hz}$ in lab air on a servo-hydraulic testing machine. Relatively thin specimens (i.e. $3 \mathrm{~mm}$ ) were used to promote a strong crystallographic growth pattern and to render surface observations representative of crack-microstructure interaction.

Both $\Delta \mathrm{P}$-controlled and $\Delta \mathrm{K}$-decreasing fatigue tests at different $\mathrm{R}$-ratios were performed to determine the material response in the Paris's regime and at near-threshold. The back-facestrain-gage technique was used to monitor continuously the crack length during the tests, [4]. Load vs. back-face-strain plots were recorded during the test in order to detect anticipated crack closure from a change in specimen compliance within the load cycle. In addition, the surface of selected specimens was polished and etched to reveal the material microstructure. Evolution of the fatigue crack through the microstructure was monitored during the tests with a microscope and digital images were periodically recorded with a CCD camera.

\section{RESULTS AND DISCUSSION}

\section{Fatigue Crack Paths}

Fatigue crack paths in a coarse-grained material become complicated even at relatively high stress intensities. The resulting crack tortuosity involves several mechanisms and tends to reduce the effective stress intensity range, $\Delta \mathrm{K}_{\text {eff }}$, below the nominally applied range, $\Delta \mathrm{K} . \mathrm{A}$ summary of the main mechanisms observed in tortuous cracks is given in Fig. 1a, [5]. RICC limits the minimum stress intensity, hook or 'lock-up' mechanisms limit the maximum stress 
intensity and branching produces true elastic shielding, [1]. Tortuosity also increases the ratio of the true length to that projected on the plane of the stress axis reducing the energy release rate.

An example of a crack propagated in Mg-alloy AZ91 at near-threshold conditions is shown in Fig. 1b: it demonstrates that both micro- and macro-roughness are observed in this coarsegrained material as well as crack deflections. The crack branching mechanism was also observed even if it is not visible in Fig. $1 \mathrm{~b}$.

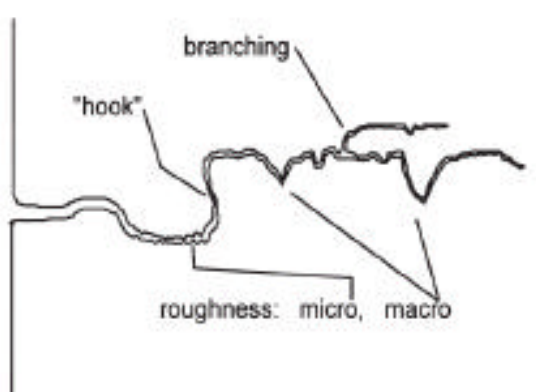

a)

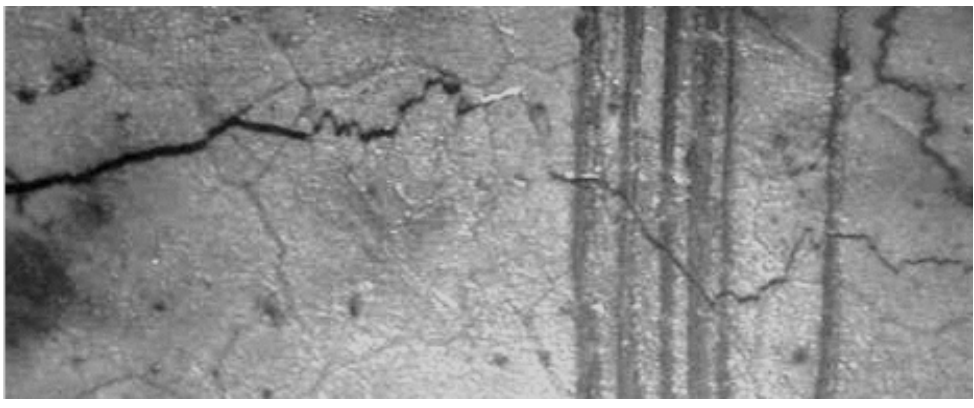

b) $\quad 100 \mu m$

Figure1. a) scheme of possible mechanisms attending near-threshold fatigue crack growth, [5] b) magnified view of crack path in coarse-grained magnesium alloy.

The crack path of Fig. $1 \mathrm{~b}$ is the result of a series of mechanisms associated with different stages of fatigue crack growth that are now discussed with the help of crack path details. When the plastic zone extends over a number of grains due to a high $\Delta \mathrm{K}$ or to a fine-grained material, quasi continuum mechanisms are operative. A rectilinear Mode I crack path, also termed a Stage II fatigue crack, occurs during fatigue crack propagation of long cracks as in Fig. 2a. A dual-slip system is active at the crack tip, (see scheme of Fig. 2b), and the crack growth process involves simultaneous or alternating flow along this slip system.

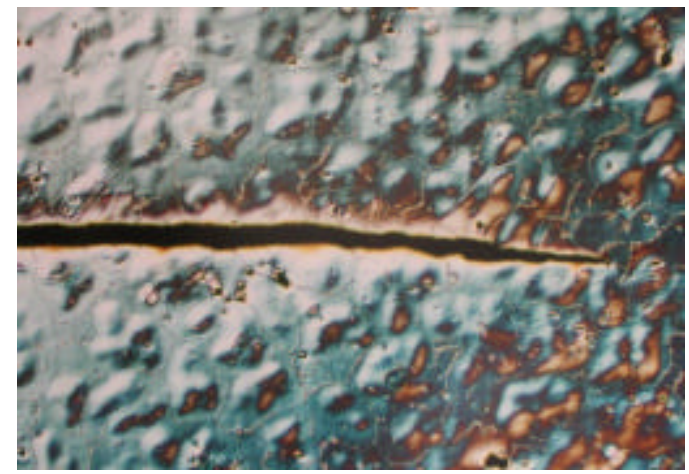

a)

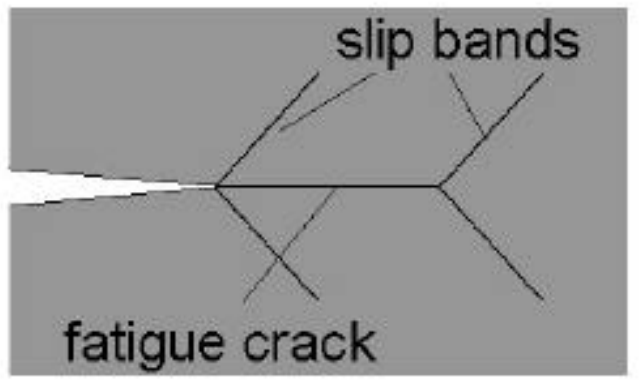

b)

Figure 2 - a) Fatigue crack growth in stage II in AZ91 and b) dual-slip band model.

When the growth rates are reduced toward threshold conditions specific features can be observed. A Stage I crack growth, occurring predominantly by single shear in the direction of the primary slip system, can develop because the local plastic zone becomes smaller than the average grain size. Fig. 3a shows a typical crack path across a few grains in the AZ91 Mgalloy. Crack propagation is characterized by crack deflection from grain to grain as in the scheme of Fig. 3b. The crack growth rates also change going through the grain boundary. A zigzag path is obtained resulting in a rough fracture surface with a faceted cleavage-like appearance. This mechanism activates crack tip shielding due to mixed-mode loading and the resulting rough fracture surface contributes to the RICC mechanism. 


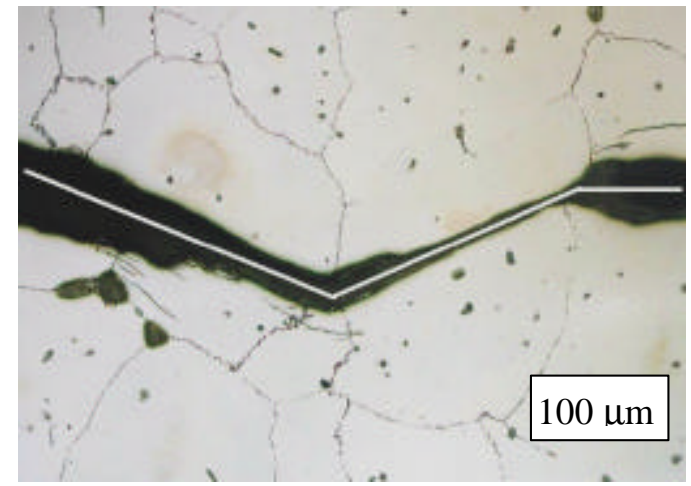

a)

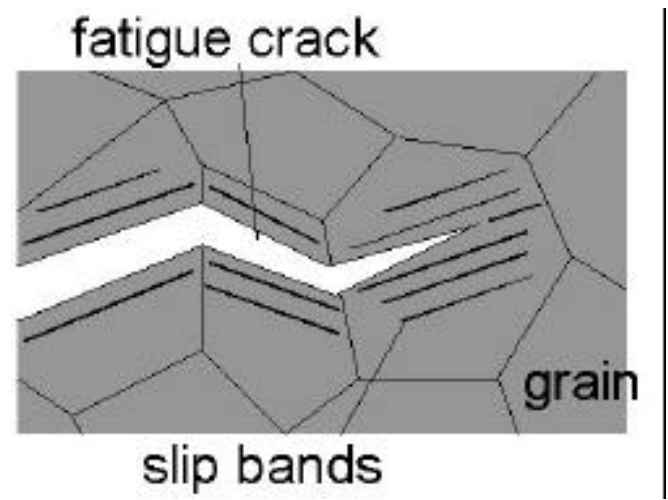

b)

Figure 3. a) Stage I growth in AZ91 and single-slip model with grain-to-grain deflection.

In the case of a very coarse grain structure, the plastic zone can be confined within a single grain even at relatively high $\Delta \mathrm{K}$. This was found in pure $\mathrm{Mg}$ and it is shown in Fig. 4a. Crack growth occurs along a deflected path, although secondary growth in directions at an angle with the main crack plane further dissipates crack tip energy. A saw-tooth type path is obtained within the grain. A possible model is depicted in Fig. $4 \mathrm{~b}$.

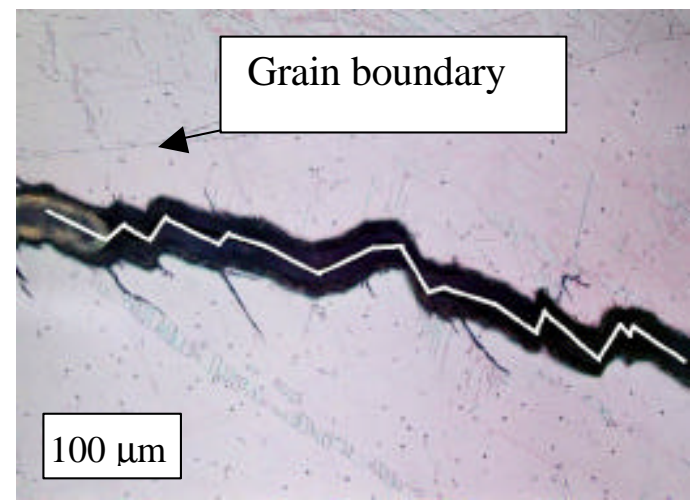

a)

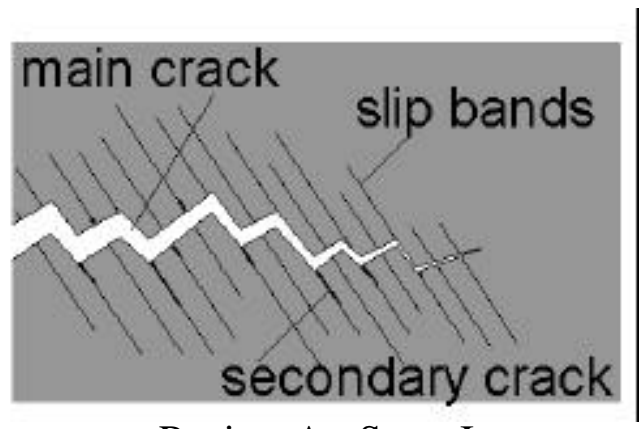

Regime A - Stage I

b)

Figure 4. a) micro zigzag path within a single grain of pure $\mathrm{Mg}$ and associated model.

\section{Fatigue Crack Growth Rates}

In this work only the near-threshold fatigue crack growth test results for the Mg-alloy AZ91 are shown in Fig. 2a and discussed. Two R-ratios were considered to assess the role of closure mechanisms and the respective data sets are found to be shifted one with respect to the other indicating that closure-type mechanisms are indeed active at low $\Delta \mathrm{K}$. Both data sets show also some scatter but it was expected as the tests were performed on relatively thin specimens with a coarse microstructure. It will be shown in a subsequent section that crack growth through the microstructure involved significant rate irregularities, which show up also in the present plot.

It is known that low R-ratios and near-threshold conditions may favor the development of RICC and oxide-induced crack closure (OICC) mechanisms rather than Elber's plasticityinduced crack closure (PICC), [1,5]. Therefore crack opening load levels during the fatigue tests were determined according to the practice of ASTM E-647 from the specimen compliance loop in a loading cycle. 


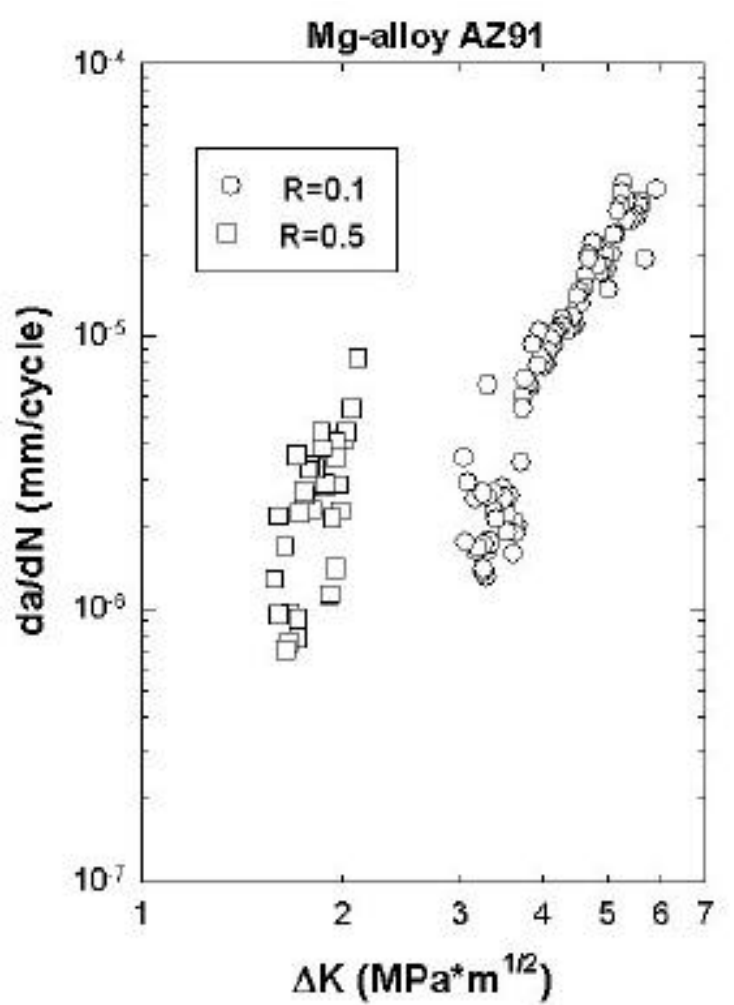

a)

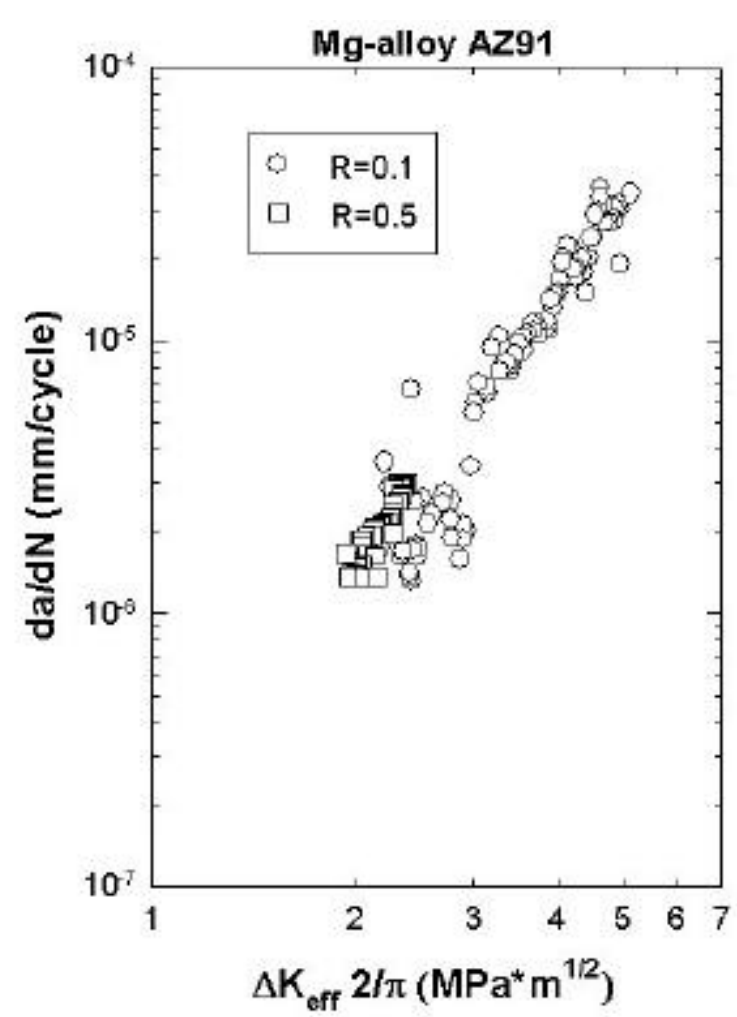

b)

Figure 5. Near-threshold fatigue crack growth rates in Mg-alloy AZ91 a) nominal $\Delta K$, b) effective $\Delta \mathrm{K}$ according to the partial crack closure model.

As recently pointed out by Donald, [6], however, the ASTM technique may provide misleading crack closure information in the near threshold regime. Donald has proposed an alternate method, the 'Adjusted Compliance Ratio', ACR, for measuring the effective stress intensity range [6,7]. It corrects the applied stress intensity range, $\Delta \mathrm{K}$, with the ratio of the measured average compliance and the compliance in the absence of closure. The effective $\Delta \mathrm{K}, \Delta \mathrm{K}_{\mathrm{eff}}$, i.e. the range in which the crack is open is defined as

$$
\Delta \mathrm{K}_{\mathrm{eff}}=\Delta \mathrm{K}^{*} \mathrm{ACR}
$$

where $A C R=\left(C_{s}-C_{i}\right) /\left(C_{o}-C_{i}\right)$ and $C_{i}$ is the specimen compliance before crack initiation, and $C_{s}$ e $\mathrm{C}_{\mathrm{o}}$ are obtained from the load vs. compliance plot for a cracked specimen. $\mathrm{K}_{\mathrm{open}}$ can then be obtained as

$$
\mathrm{K}_{\mathrm{open}}=\mathrm{K}_{\max }-\Delta \mathrm{K}_{\mathrm{eff}}
$$

However, the partial closure model, recently advanced in [2], was also adopted to obtain the good correlation (independent of R-ratio) of Fig 5b. The model was developed after recognizing that a significant contribution to fatigue damage occurs in the load range below the opening load as measured by compliance techniques. This so-called "Donald" effect, [7], is considered especially relevant at near-threshold growth rates and it is based on the idea that OICC and RICC mechanisms occur at a distance behind the crack tip. Surface roughness and oxide layers act like a wedge inserted between the crack surfaces and crack tip damage may occur even when $\mathrm{K}<\mathrm{K}_{\mathrm{open}}$, i.e. partial crack closure. Paris [2] showed that when closure is 
modeled as being due to an elastic wedge the effective opening load is only about $2 / \pi$ times its value as customarily measured. That is

$$
\Delta \mathrm{K}_{\mathrm{eff}}=\mathrm{K}_{\max }-(2 / \pi) \mathrm{K}_{\mathrm{open}}
$$

This is the parameter used to replot in Fig. 5b the data for near-threshold fatigue crack growth in Mg-alloy AZ91 of Fig. 5a. A linear trend common to the two data sets in the entire range of growth rates is found. Support to this model has been obtained from tests in different steels and will be presented shortly, [8].

\section{Fatigue Crack Growth through a Microstructure}

In this section observations of the fatigue crack path - microstructure interaction are presented in terms of images of etched microstructures and corresponding crack growth rates as measured by the crack length vs. compliance technique, [4]. As discussed before crack tortuosity has a 'shielding' effect by reducing the effective stress intensity range by crack deflection and by promoting roughness induced crack closure. The first observation is shown in Fig. 6 and is for pure $\mathrm{Mg}$.
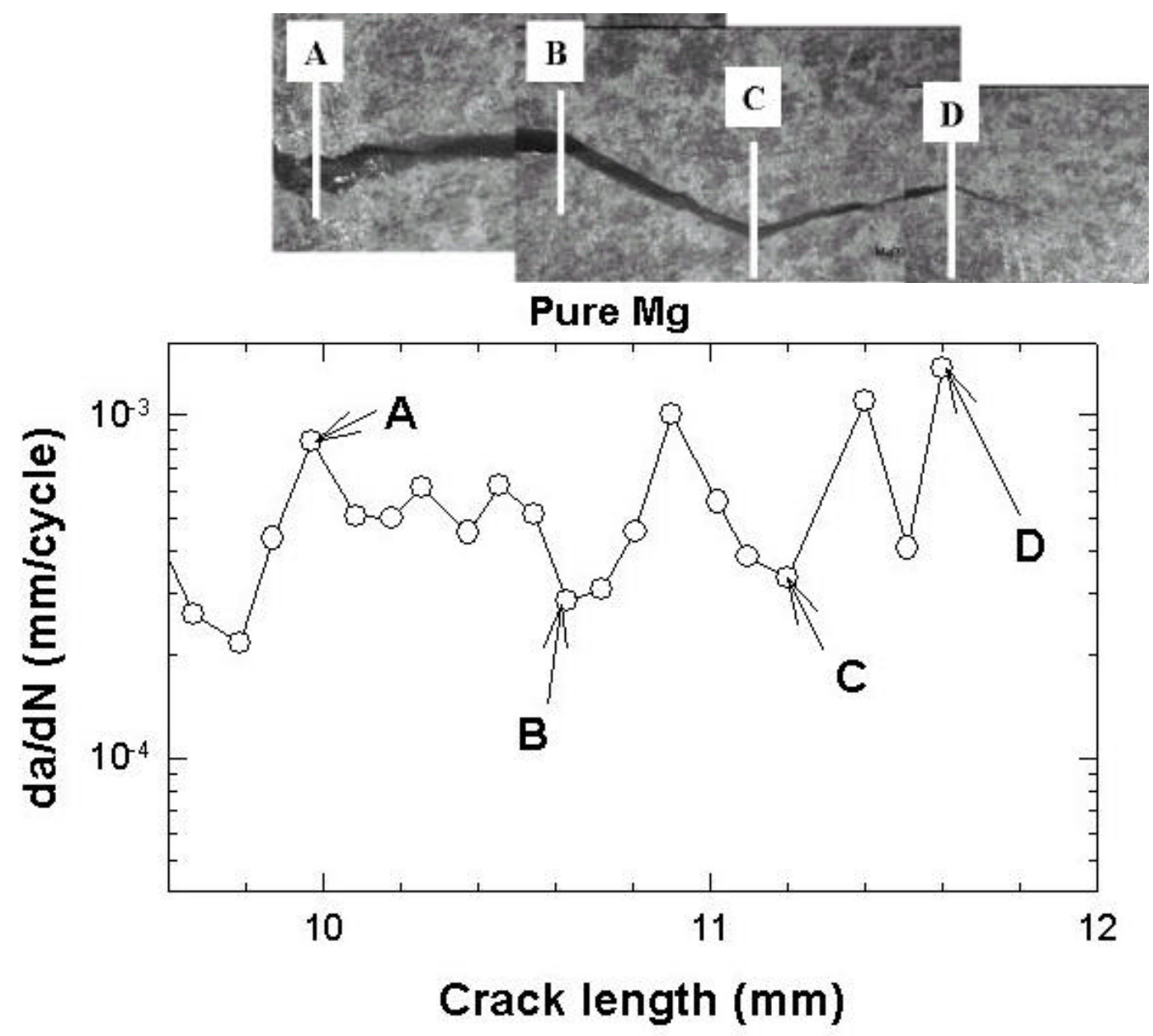

Figure 6. Magnified view of crack path in pure $\mathrm{Mg}$ and growth rate variations during propagation through the microstructure.

Figure 6 shows on top a magnified fatigue crack presenting a well-defined growth along crystallographic planes with deflections occurring at grain boundaries. In the plot of Fig. 6 letters 
show corresponding locations along the crack and specific growth rates as measured during continuous optical monitoring of the crack. It is seen how the crack accelerates before reaching a grain boundary where it decelerates because it behaves like an obstacle. Small cracks have been also observed to arrest at grain boundaries, [9], and growth rates have shown minima when the size of the plastic zone was approximately equal to that of the grain, [10]. Tanaka et al., [10], proposed the blocked slip band concept, in which transference of the slip band from one grain into the next controls the rate of propagation. Although the present cracks are long, the coarse grain size of the materials apparently significantly affects the fatigue crack growth rates.

In addition to crack deflections and grain boundaries, other phenomena may affect crack velocity and path direction. In Fig. 7 a fatigue crack growing in the Mg-alloy AZ91 is shown. In the image the crack path is observed to pass between two dark circular areas. They are intermetallic compound $\mathrm{Mg}_{17} \mathrm{Al}_{12}$ particles that have a lower strength than the surrounding material and are incompatible with the magnesium structure. These particles have been found to influence the crack path. The example of Fig. 7 shows how the crack initially deflected when it approaches the particles becomes rectilinear and propagates between them. From the point of view of crack velocity the crack accelerates within the grain before reaching point $\mathrm{A}$.
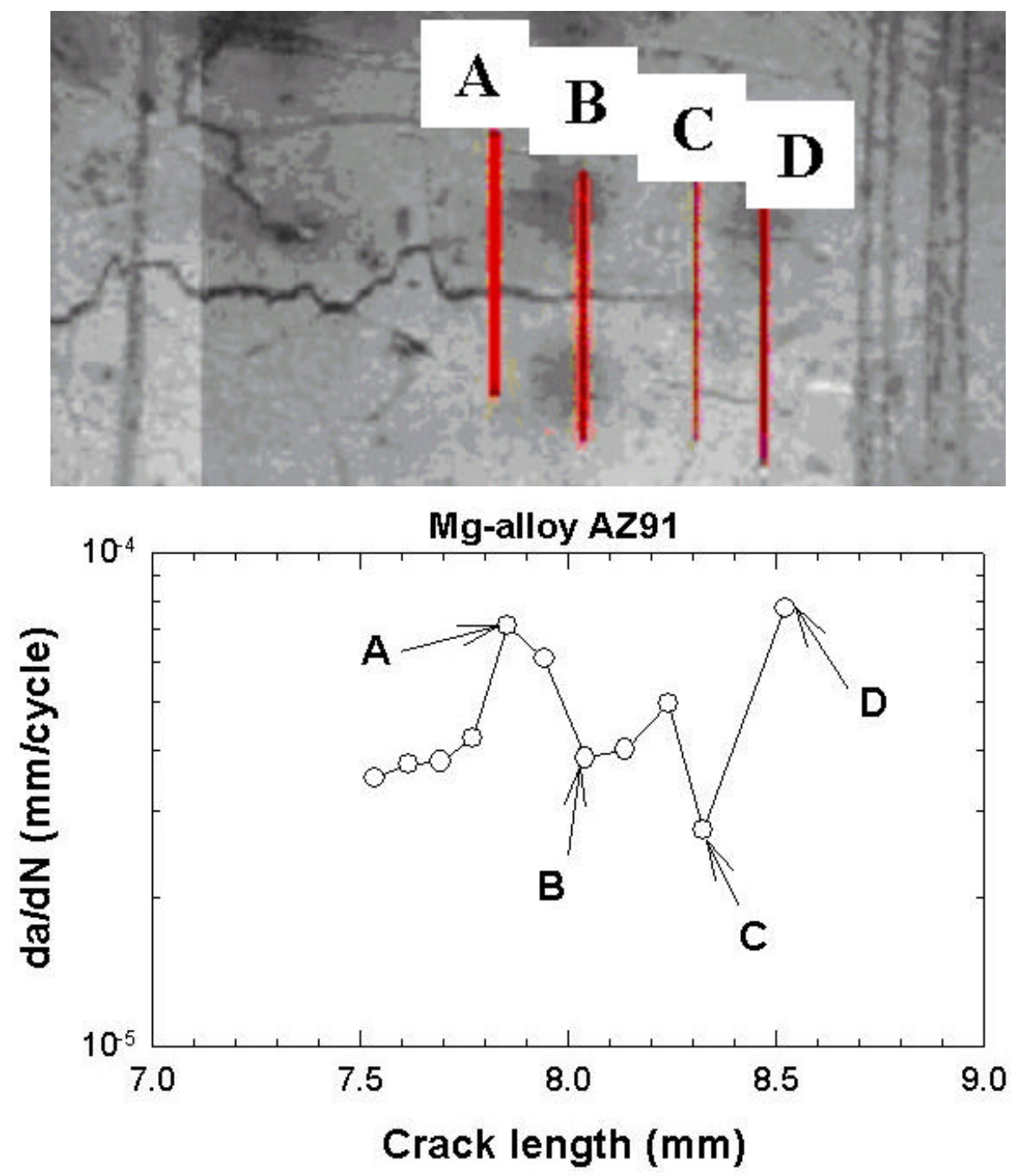

Figure 7. Magnified view of crack path in Mg-alloy AZ91 and growth rate variations during propagation through the microstructure. 
Then from point $\mathrm{A}$ to point $\mathrm{C}$ the velocity decreases with an oscillation due to a change in crystalline plane at a grain boundary not visible in the picture. This effect could be due to a residual stress field in the vicinity of the particles. After point $\mathrm{C}$ the crack rapidly accelerate toward point $\mathrm{D}$.

\section{CONCLUSIONS}

Fatigue crack growth tests in coarse-grained Mg-alloys were examined from the point of view of near-threshold behavior and of crack path-microstructure interaction by monitoring optically the surface of etched specimens. The main conclusions of this study are as follows:

a Near-threshold fatigue crack growth promotes the development of rough surfaces by activation of single slip mechanisms and crack deflection.

a Roughness-induced crack closure was invoked to address the R-ratio effect on fatigue crack growth rates.

a The partial crack closure model, based on the assumption that the crack is always open for a length of the order of a tenth of micron behind the tip, was successful in correlating fatigue crack growth rates.

a Monitoring optically cracks growing through the microstructure revealed that growth rates are affected by grain boundaries and grain-to-grain crack deflections;

\section{REFERENCES}

1. Suresh, S. (1998) Fatigue of Materials, $2^{\text {nd }}$ Ed., Cambridge University Press, UK.

2. Paris, P., Tada, H. and Donald, J.K. (1999) Int. J. Fatigue 21, pp. 35-46.

3. Mordike, B.L. and Ebert, T. (2001) Mat Sci Eng A302, 37-45.

4. Nicoletto, G. and Pirondi, A. (1997) Materialove inzinierstvo 4, Zilina, Slovakia, 10-16.

5. Lawson, L., Chen, E.Y. and Meshii M. (1999) Int J. Fatigue 21, S15-S34.

6. Donald, K. (1997) Int. J. Fatigue 19, S191-S195.

7. Donald, K. and Paris, P. (1999) Int. J. Fatigue 21, 47-57.

8. Fersini, D. and Nicoletto, G. (2003) Procs. TRANSCOM Conference, University of Zilina, Slovakia (to appear).

9. Taylor, D. (1990) Fatigue thresholds, Butterworth, UK.

10. Tanaka, K., Nakai, Y. and Yamashita, M. (1981) Int. J. Fatigue 17, 519-533. 